\title{
Caracterización del suicidio y su incidencia en la provincia de Tungurahua: estudio descriptivo y análisis estadístico
}

Fecha de recepción: 2021-09-24 • Fecha de aceptación: 2021-11-23 • Fecha de publicación: 2022-01-10

\author{
Jorge Oswaldo Tamayo Viera' \\ Tecnológico Superior Universitario España, Ecuador \\ jorge.tamayo@iste.edu.ec \\ https://orcid.org/0000-0002-2554-8684 \\ Vicente Leonardo Pérez Yauli² \\ Estudio Jurídico Acosta \& Asociados, Ecuador \\ p.leonardo94@yahoo.es \\ https://orcid.org/0000-0003-1937-7820 \\ Ibeth Aracelly Molina Arcos ${ }^{3}$ \\ Tecnológico Superior Universitario España, Ecuador \\ ibetha.molinaa@iste.edu.ec \\ https://orcid.org/0000-0001-9650-1317 \\ Edison Manuel Arroba Freire ${ }^{4}$ \\ Tecnológico Superior Universitario España, Ecuador \\ edison.arroba@iste.edu.ec \\ https://orcid.org/0000-0002-7196-0784
}

\section{Resumen}

En la presente investigación se propuso como objetivo efectuar un análisis del suicido y de las causas que lo motivan en el contexto de la provincia de Tungurahua. Los métodos de investigación que fueron utilizados refieren al cualitativo necesario para la identificación de las cualidades del objeto de estudio; cuantitativo empleado para desarrollar la parte estadística la cual consistió en la recolección de datos, análisis e interpretación en relación con los datos proporcionados por 
el Ministerio del Interior, Dirección Nacional de Investigación de Delitos contra la Vida, Muertes Violentas, Desapariciones, Secuestros y Extorsión, Instituto Nacional de Estadística y Censos; descriptivo utilizado para enunciar las características del objeto estudiado. El suicidio a nivel mundial, de Latinoamérica, Ecuador y de la provincia de Tungurahua se ha incrementado de forma acelerada, cuya ocurrencia se presenta con mayor incidencia en la población comprendida entre la adolescente y juventud, obedeciendo a causas de origen psicológico, familiar, social y cultural. El periodo de estudio se centró entre los años 2014 - 2021, en la provincia de Tungurahua.

\title{
Palabras clave: suicidio, adolescencia, comportamiento suicida, problemas, influencia
}

\begin{abstract}
In the present investigation, it was proposed to carry out an analysis of the suicide and the causes that motivate it in the context of the province of Tungurahua. The research methods that were used refer to the qualitative necessary for the identification of the qualities of the study object; quantitative employee to develop the statistical part which consisted of the collection of data, analysis and interpretation in relation to the data provided by the Ministry of the Interior, National Directorate of Research for Crimes against life, violent deaths, disappearances, kidnappings and extortion, National Institute of Statistics and Censuses; descriptive used to enunciate the characteristics of the object studied. The suicide worldwide, in Latin America, Ecuador and the province of Tungurahua has increased accelerated, whose occurrence is presented with greater incidence in the population between adolescent and youth, obeying causes of psychological, family, social origin and cultural. The study period focused on the years 2014 - 2021, in the province of Tungurahua.
\end{abstract}

Keywords: suicide, adolescence, suicidal behavior, problems, influence 


\section{Introducción}

La investigación propuesta centro su interés en el estudio del suicidio y las causas que generan en la persona intensiones de ejecutarlo y en ciertos casos, en la consumación del mismo. Desde el punto de vista de la salud pública, el suicido es considerado un acto practicado de forma deliberada con la intensión de terminar con la vida de quien la lleva a cabo, provocándose lesiones con grado variable y utilizando diferentes objetos y medios (OMS, 2012, citado en Bedoya \& Montaño, 2016, p.79).

Los casos de suicidios producidos en el Ecuador, en sus diferentes provincias, son considerados actualmente como un grave problema que requiere la atención, tanto de instituciones públicas, como privadas, que por su actividad se ven vinculadas al área social; esto debido a los índices elevados cuantificados en los últimos años, y; la conmoción que produce en la colectividad en general y a los familiares cercanos que quien la ha practicado en particular (Miniguano, 2018, p. 4).

En la provincia de Tungurahua, la incidencia de suicidios han generado interés tanto para las autoridades relacionadas a la salud pública, como a la seguridad interna; de igual forma, existe una preocupación para las familias afectadas por su ocurrencia; a nivel nacional se han producido 977 suicidios en las diferentes provincias; la circunstancias para la consumación de estos hechos se relacionan con problemas sentimentales, familiares entre las más principales (Salgado, 2020).

El suicido a nivel mundial alcanza un índice del $60 \%$, lo cual advierte, que el número de defunciones por esta práctica llega casi el millón de muertes, estos datos se traducen en una tasa de mortalidad de dieciséis personas fallecidas por cada 100.000 habitantes, de acuerdo con estos datos, una persona fallece por suicidio cada cuarenta segundos en algún lugar del planeta (Echávarri et al., 2015, p.3).

De acuerdo con lo indicado, por lógica es entendible que este fenómeno lejos de disminuir con el trascurso del tiempo, se ha incrementado y a paso acelerado; esta práctica comúnmente es llevada a cabo en la adolescencia, etapa del desarrollo humano considerada bastante conflictiva y de riesgo, debido a la influencia que reciben del entorno en el que se desenvuelven, lo cual afecta el comportamiento y estado de salud de la persona; el suicidio un vez que ha sido consumado afecta al entorno familiar y social de la víctima (Cuesta Revé, 2017, p. 72).

De forma general, los métodos empleados para llevar a cabo el suicidio dependen en mucho de la facilidad y disponibilidad de acceso de la persona a estos; además de los antecedentes familiares en la utilización de un determinado método(s), lo cual depende en gran medida de la influencia social y cultural, como también de las características geográficas que circundan al sujeto; como la cercanía al mar, vías férreas, pesticidas, puentes, carreteras bastante transitadas, edificios altos, ahogamiento, intoxicación con medicamentos y gases, envenenamiento con diferentes elementos, inanición, utilización de armas de fuego o corto punzantes, ahorcamiento, precipitación, etc (Cortés, 2019, p. 14).

Los factores que interactúan para inducir a una persona a concebir pensamientos y comportamientos suicidas son variados; entre estos se encuentran los psicológicos, familiares, 
sociales y culturales; no obstante, que los estudios científicos aportan datos alentadores en la prevención de las practicas suicidas, el tratamiento de esta problemática como política prioritaria de los distintos gobiernos ha tenido escasa prioridad, centrando su interés en otro tipo de conflictos (Organización Panamericana de la Salud, 2014).

Entre las causas psicológicas que con mayor frecuencia inducen al suicidio se encuentran, los trastornos psicológicos, ansiedad, depresión, déficit de atención, consumo de bebidas alcohólicas y sustancias prohibidas, constantes problemas de salud (hospitalización), etc., cuyo origen se encuentra en la genética de las personas, en la historia familiar y/o abusos sufridos en la infancia.

Las causas familiares comúnmente comprenden conflictos familiares y la desorganización, abuso sexual, ausencia de uno o de ambos progenitores, separación familiar (divorcio - migración), abandono, aislamiento familiar y social, falta de atención y comunicación, etc., factores que encuentran su procedencia en hogares desorganizados, en los que el maltrato físico se ha vuelto una práctica común entre sus miembros (Corona, 2016 citando en Miniguano, 2018, p. 51).

Entre las causas sociales se encuentran la falta de oportunidades laborales, desempleo, problemas socio - económicos, disgregación social, escaso nivel educativo, pobreza, dificultad de acceso a los servicios de salud, discriminación, violencia, etc., cuya raíz se encuentra generalmente en las políticas y decisiones adoptadas por los gobiernos de turno.

Las causas culturales que influyen en la generación de pensamientos suicidas comprenden, origen de la persona, raza, color, etnia, creencias, orientación sexual, inseguridad, etc., cuya procedencia se encuentra en las relaciones interpersonales de la cotidianidad de las personas.

La literatura sobre el suicidio afirma que muchos de estos se producen de forma impulsiva; es decir, como respuesta a las crisis que la persona, familia, sociedad, tienen que afrontar mermando las capacidades de la persona para confrontar las situaciones conflictivas propias de la vida, entre estas, problemas financieros, rupturas de relaciones, enfermedades (Organización Mundial de la Salud, 2019).

Los modelos de suicidio, de acuerdo con Pérez (2019), se presentan en tres categorías:

- Subjetivo, ejecutado tras una evaluación de la realidad circundante del sujeto, relacionado a estadios de depresión, abuso en el consumo de sustancias prohibidas.

- Objetivo, ateniente a situaciones ciertas y verificables como la violencia de género, hecho tras el cual la víctima es afectada física y psicológicamente, concibiendo como única forma de salida el suicidio.

- Mixto, el cual constituye una combinación de la primera y segunda categoría.

Los estudios sobre el suicidio lo han clasificado en dos grandes grupos, que se ven descritos en la Tabla 1: 
Tabla 1.

Clasificación del suicidio

\begin{tabular}{ll}
\hline Grupo & \multicolumn{1}{c}{ Definición } \\
\hline \multirow{3}{*}{ Origen } & $\begin{array}{l}\text { Psicótico. Percepción ajena al entorno y la realidad. } \\
\text { Neurótico. Falta de comprensión real del suicidio. } \\
\text { Psicodisplásico. Relacionado con la psicopatía y } \\
\text { agresividad. } \\
\text { Filosófico. Vacío existencial del sujeto. }\end{array}$ \\
\hline \multirow{2}{*}{ Forma } & $\begin{array}{l}\text { Impulsivo. Inmediatez en los actos del sujeto. } \\
\text { Obsesivo. Constantes ideas de autodestrucción. } \\
\text { Reflexivo. Frecuente en los sujetos con depresión. }\end{array}$ \\
\hline
\end{tabular}

Acorde a lo señalado con anterioridad, y de acuerdo con la Organización Mundial de la Salud, Ecuador ocupa el cuarto lugar dentro de la clasificación de las tasas de suicido a nivel de Latinoamérica, lo cual corresponde a un porcentaje de $13.1 \%$ por cada 100.000 habitantes (Cazar, 2014, citado en Almeida, 2017, p. 4).

En relación con el grupo poblacional en el cual el suicidio tiene mayor incidencia (adolescentes - jóvenes), los estudios señalan que en determinados países los índices a nivel mundial son elevados, tal es el caso de Ecuador, en el que durante las últimas dos décadas, alrededor de 300 jóvenes y adolescentes comprendidos entre los 10 y 24 años se quitaron la vida anualmente (Gerstner et al., 2019).

Tabla 2.

Estadísticas de los suicidios por grupo de edad en Tungurahua

\begin{tabular}{ccccccc}
\hline Grupo de edad & $\mathbf{2 0 1 4}$ & $\mathbf{2 0 1 5}$ & $\mathbf{2 0 1 6}$ & $\mathbf{2 0 1 7}$ & $\mathbf{2 0 1 8}$ & $\mathbf{2 0 1 9}$ \\
\hline 5 a 9 años & 0 & 0 & 0 & 0 & 0 & 1 \\
\hline 10 a 14 años & 1 & 7 & 5 & 5 & 4 & 3 \\
\hline 15 a 19 años & 2 & 5 & 11 & 6 & 14 & 13 \\
\hline 20 a 24 años & 10 & 11 & 10 & 6 & 11 & 14 \\
\hline 25 a 29 años & 3 & 7 & 7 & 12 & 8 & 6 \\
\hline 30 a 34 años & 3 & 6 & 9 & 7 & 8 & 1 \\
\hline 35 a 39 años & 3 & 2 & 5 & 2 & 4 & 8 \\
\hline 40 a 44 años & 4 & 5 & 2 & 1 & 7 & 6 \\
\hline 45 a 49 años & 2 & 1 & 1 & 2 & 2 & 3 \\
\hline 50 a 54 años & 1 & 1 & 2 & 4 & 1 & 0 \\
\hline 55 a 59 años & 1 & 1 & 4 & 1 & 2 & 3 \\
\hline 60 a 64 años & 2 & 6 & 4 & 0 & 1 & 1 \\
\hline 65 a 69 años & 0 & 1 & 2 & 3 & 2 & 0 \\
\hline 70 a 74 años & 2 & 2 & 1 & 4 & 2 & 0 \\
\hline 75 años y más & 0 & 2 & 1 & 0 & 4 & 3 \\
\hline
\end{tabular}

Fuente: Instituto Nacional de Estadística y Censos (2020) 
De las defunciones externas, la provincia de Tungurahua registra un total de doce muertes violentas que representan el $58,3 \%$ a los hombres y el $41,7 \%$ a las mujeres. Seis fallecidos corresponden a otros accidentes de transporte, cinco por accidentes de transporte terrestre y un caso de suicidio registrado en el grupo de edad de los cinco a nueve años en el 2019. El mayor número de suicidios se presenta en el rango comprendido entre los 15 a los 29 años de edad durante los años 2018 y 2019 , como se evidencia en la Tabla 2.

Tabla 3.

Levantamiento según tipo de muerte Sub zona 18 Tungurahua

\begin{tabular}{ccc}
\hline Tipo de muerte & No. Fallecidos & Porcentaje \\
\hline Homicidio & 1 & $1,14 \%$ \\
\hline Asesinato & 5 & $5,68 \%$ \\
\hline Suicidio & 21 & $23,86 \%$ \\
\hline Fortuito/fuerza mayor & 38 & $43,18 \%$ \\
\hline Natural & 18 & $20,45 \%$ \\
\hline Feto & 3 & $3,41 \%$ \\
\hline Aborto & 1 & $1,14 \%$ \\
\hline Osamenta & 1 & $1,14 \%$ \\
\hline Total & 88 & $100,00 \%$ \\
\hline
\end{tabular}

Fuente: Ministerio del Interior (2017)

En la sub zona 18 de la Policía Nacional del Ecuador, al finalizar el mes de mayo del 2017 se registraron en Tungurahua un total de ochenta y ocho casos de personas fallecidas por diferentes motivos (ver Tabla 3), como causa natural o por muertes violentas, siendo los suicidios la segunda causa de muerte, con un $23,86 \%$, según información de la Dirección Nacional de Delitos contra la vida, muertes violentas, desapariciones, extorsión y secuestros.

\section{Metodología}

En la ejecución de la presente investigación se ha utilizado el método cualitativo para la identificación del objeto de estudio, para esto se tomaron de referencia estudios relativos al tema, y realizados por diferentes autores y organismos; cuantitativo en el análisis de datos recopilados, contando para ello con el aporte significativo de entidades gubernamentales, mismos que fueron representados mediante tablas estadísticas; descriptivo necesario para caracterizar al objeto de estudio y establecer su clasificación.

La investigación comprendió el periodo 2014 - 2021, de acuerdo a la información aportada por el Ministerio del Interior, Dinased (Dirección Nacional de Investigación de Delitos contra la Vida, Muertes Violentas, Desapariciones, Secuestros y Extorsión), Instituto Nacional de Estadística y Censos (INEC), dentro de la provincia de Tungurahua y los distintos cantones que componen esta circunscripción territorial. 


\section{Resultados}

La provincia de Tungurahua presentó cifras considerables de casos de suicidios dentro de los años de análisis. Su incremento fue progresivo en los meses de septiembre, octubre y diciembre. El rango comprendido entre los 15 a los 29 años, registra las mayores estadísticas de lesiones autoinflingidas intencionalmente. Los medios más utilizados para el suicidio son las cuerdas, cables, correas, prendas textiles, constituyéndose el distrito Ambato sur el sector en el que se registran, la mayor cantidad de casos de suicidios, de acuerdo a la información estadística proporcionada por el Ministerio del Interior del Ecuador.

\section{Figura 1.}

Registro total de lesiones autoinflingidas intencionalmente en el país

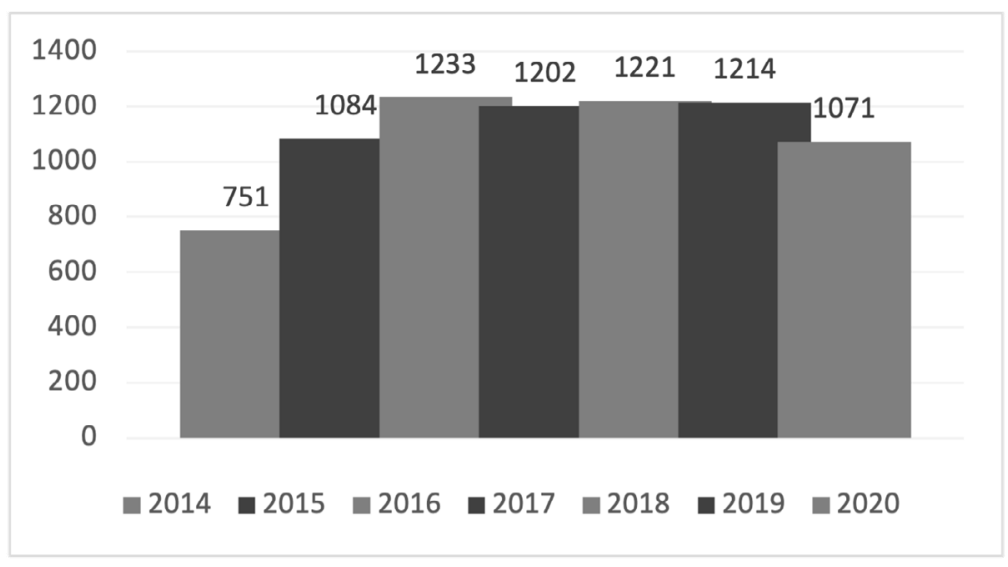

Fuente: Instituto Nacional de Estadística y Censos (2020)

A nivel nacional, de acuerdo a la información proporcionada por la Dirección Nacional de Delitos contra la Vida, Muertes Violentas, Desapariciones, Extorsión y Secuestros, en el año 2016 se registran mil doscientos treinta y tres casos de suicidios, siendo el mayor número de lesiones autoinflingidas intencionalmente, seguido del año 2018 con mil doscientos veinte y un reporte de todas las provincias del país, como se ve en la Figura 1. 
Figura 2.

Registro total de lesiones autoinflingidas intencionalmente en Tungurahua

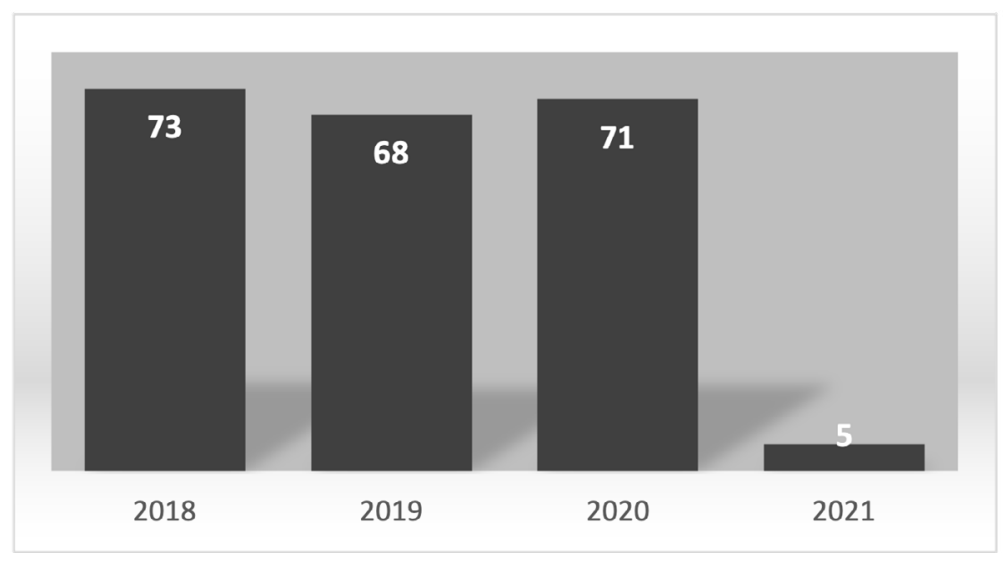

Fuente: Moreta (2021)

De acuerdo con la Figura 2, durante los meses de enero y febrero del año 2021 se registran cinco casos de suicidios en la provincia de Tungurahua. En el año 2018 se presenta una buena incidencia de lesiones autoinflingidas intencionalmente con setenta y tres reportes. En el 2020 se contabilizan setenta y un casos y en el año 2019, el informe proporcionado por la Dinased menciona un total de sesenta y ocho suicidios.

Figura 3.

Suicidios por mes en la provincia de Tungurahua

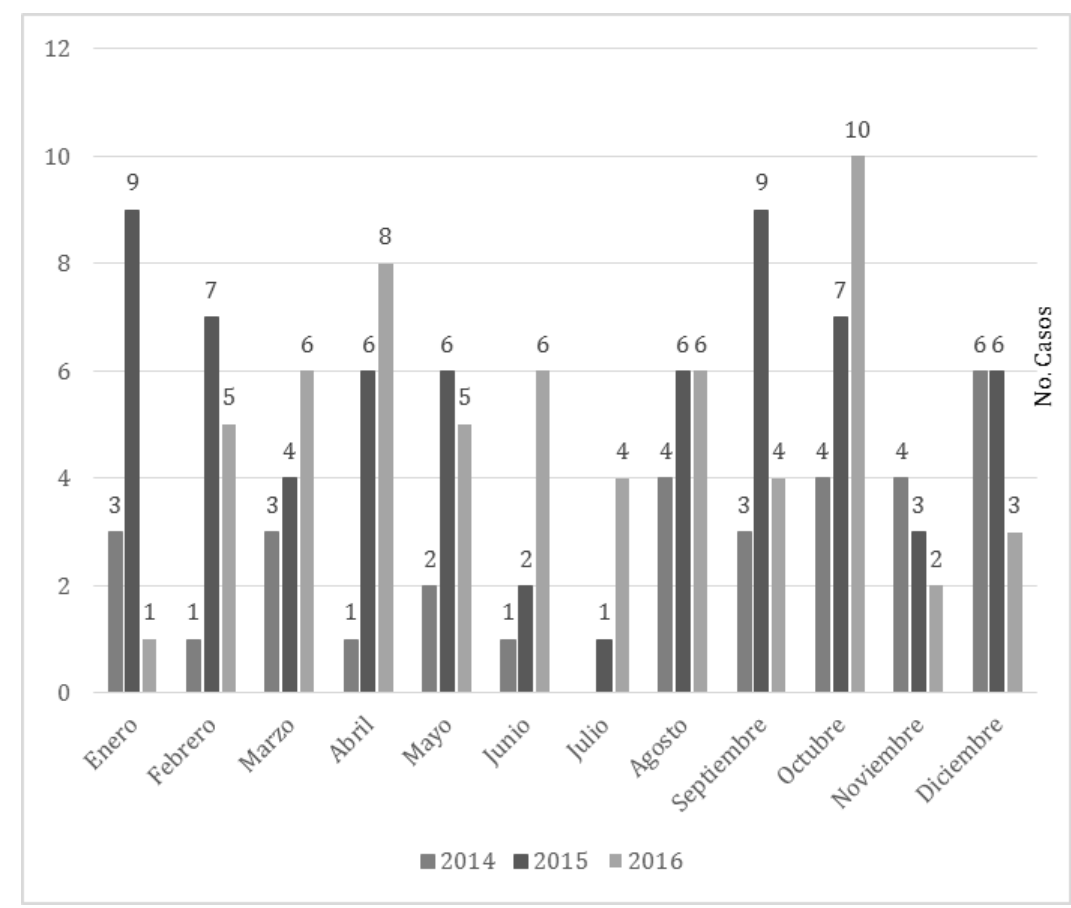

Fuente: Ministerio del Interior (2017) 
Además, se evidencia que en el mes de diciembre el mayor número de suicidios para el año 2014 con seis casos, en el año 2015, durante los meses de enero y septiembre se presentaron nueve casos. El mes de octubre del año 2016 la incidencia de suicidios fue mayor con diez casos (Figura 3), de igual manera a ese mes le corresponde el 13,29\% con veintiún casos de suicidios presentados en los años 2014, 2015 y 2016.

Figura 4.

Suicidios por distrito en la provincia de Tungurahua

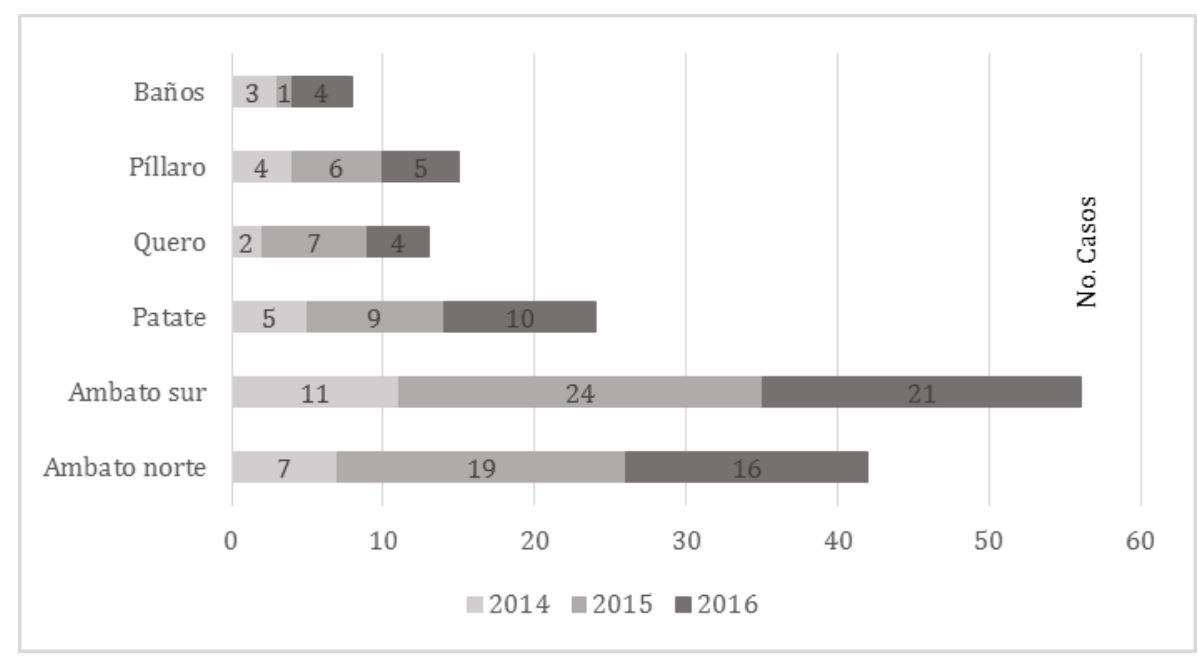

Fuente: Ministerio del Interior (2017)

La mayor incidencia de casos de suicidios dentro de la provincia de Tungurahua se presentó en la ciudad de Ambato, siendo el distrito Ambato sur el que registró el mayor número de suicidios con cincuenta y seis casos, como se evidencia en la Figura 4. Éstos índices equivalen al $35,44 \%$ del total de casos contabilizados en los distritos Baños, Quero, Píllaro, Patate, Ambato sur, Ambato norte, que conforman la subzona 18 Tungurahua. El distrito Ambato norte registró el $26,58 \%$ del total de casos de suicidios presentados en la provincia de Tungurahua.

En Ecuador existen ciertas entidades que llevan registros sobre los casos de suicidio; sin embargo, lo hacen con criterios que aún no están unificados, de ahí que, internamente no existe un registro oficial que dé a conocer el número y características del suicidio o intento del mismo. Tampoco hay una tradición científica, aún que indague la conducta suicida en el país y sus especificidades (Campo, 2018). 
Figura 5.

Suicidios por género en la provincia de Tungurahua

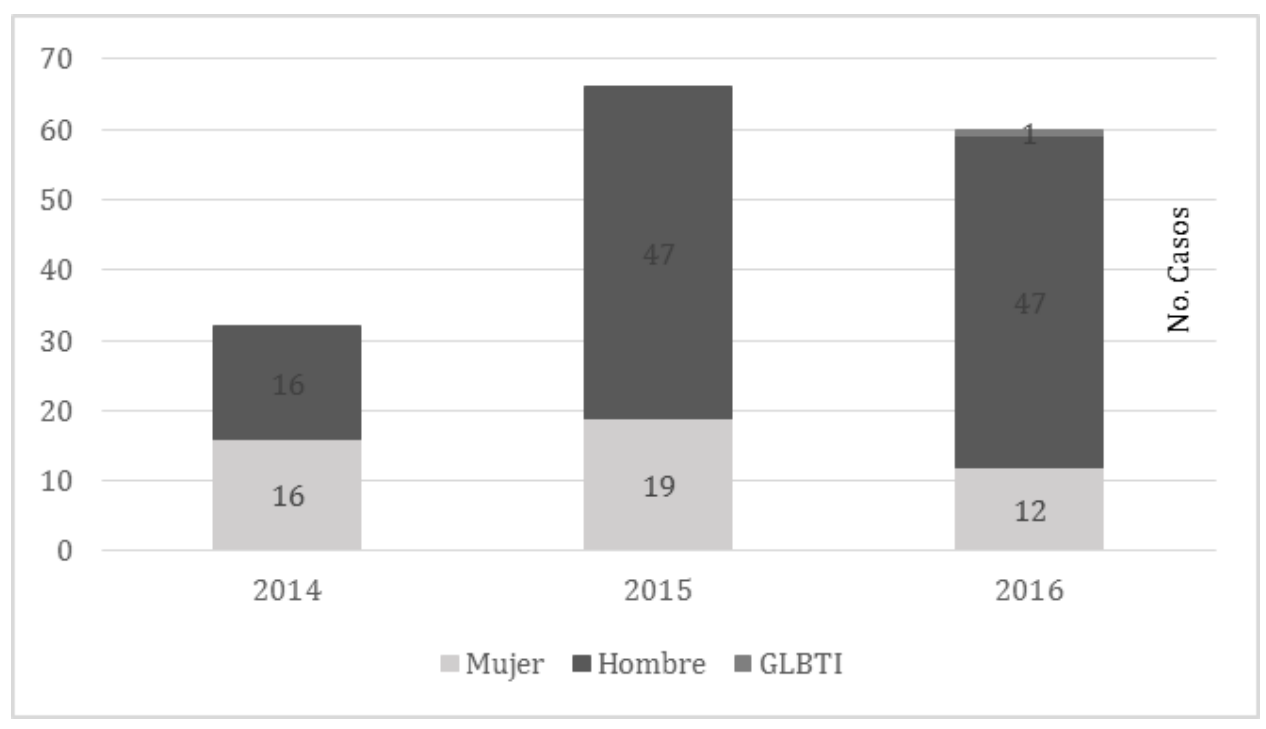

Fuente: Ministerio del Interior (2017)

Para el año 2014, de acuerdo con la Figura 5, dentro de la provincia de Tungurahua se registraron dieciséis casos de suicidios, tanto víctimas de sexo masculino, como femenino, valor que equivalente al $50 \%$ respectivamente.

En el 2015 , el $71 \%$ de suicidios en la circunscripción de estudio, correspondió a víctimas de sexo masculino y $29 \%$ a víctimas de sexo femenino, denotando una marcada diferencia con el año precedente. En este año se observa un incremento significativo de suicidios en los varones del triple de casos, que constituye un registro muy alto dentro del periodo referencial de estudio.

En el año 2016, los casos relacionados a suicidios de personas de sexo masculino se mantuvieron en igual proporcionalidad al año 2015; es decir, se registraron 47 personas que representan el $78 \%$, el $20 \%$ al sexo femenino; datos que son variantes al año anterior; incluyéndose para este año dentro del análisis a la población que corresponde al grupo GLBTI con el 2\% de casos de suicidios en la provincia de Tungurahua.

De acuerdo a los estudios efectuados a nivel mundial, Ecuador aparece entre los cinco países con las tasas más altas de suicidio, ponderando el fenómeno en mujeres adolescentes que, en hombres de su misma edad, lo cual fue confirmado por Gerstner et al. (2018), en su estudio sobre suicidio en niños y adolescentes a nivel mundial, quienes hallaron una razón hombre/mujer de 1,8:1 en el grupo de edad entre 10 y 24 años, durante el periodo 2008 a 2014. 
Figura 6.

Suicidios por edad en la provincia de Tungurahua

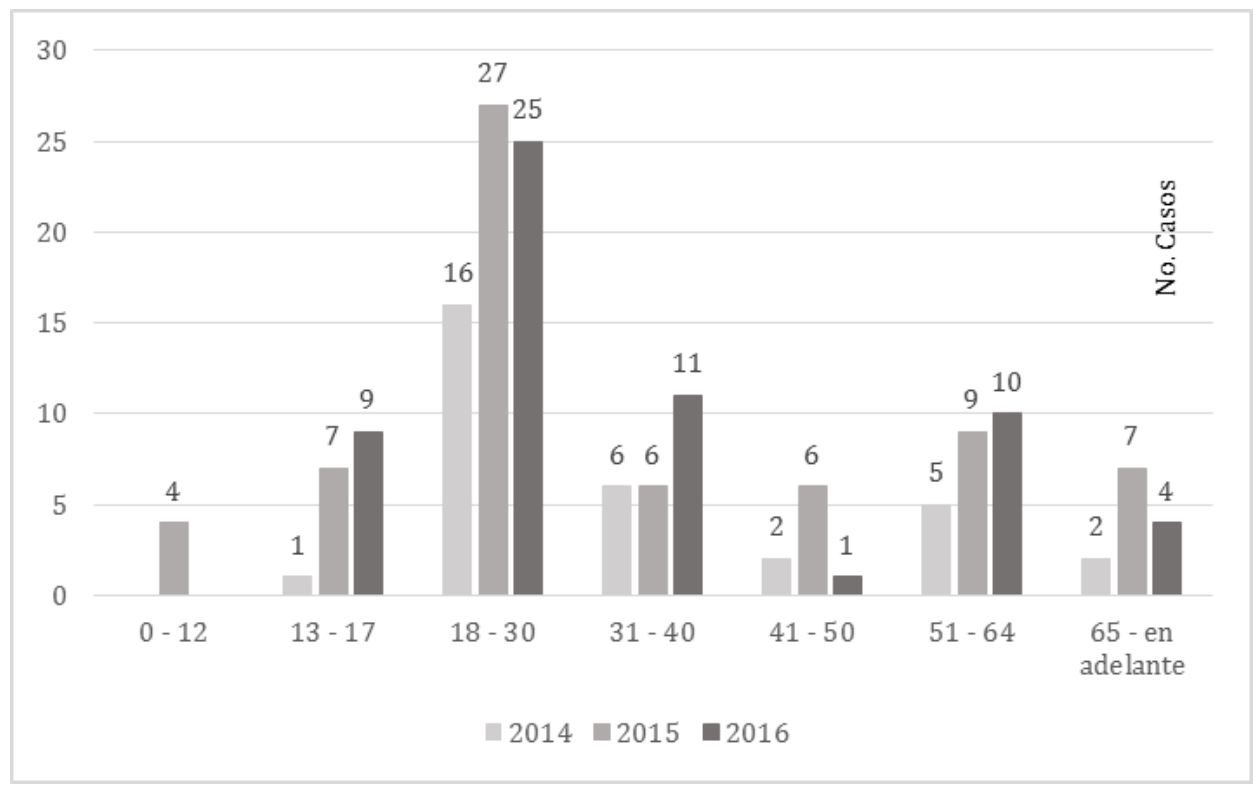

Fuente: Ministerio del Interior (2017)

Entre los años 2014 - 2016, la mayor incidencia de casos de suicidios presentados en la provincia de Tungurahua se registró en el grupo de edad comprendido entre los 18 y 30 años de edad.

Estos casos se registraron de la siguiente manera: para el año 2014, el 10,13\%; en el año 2015 el mayor número de suicidios se incrementó con 27 casos que equivalen al 17,09\%, y; para el año 2016 el porcentaje de suicidios alcanzo el 15,82\% del total de casos analizados indistintamente del sexo, condición o filiación.

El suicidio en población menor de 25 años en Ecuador es un problema destacado de salud pública, y se ha incrementado entre 1990 y 2017, especialmente, entre niños, niñas, y adolescentes y jóvenes de sexo masculino. La estrategia intersectorial propuesta en 2018 para reducir el suicido queda pendiente de evaluación e inferencia

En Ecuador, los índices de suicidio se han incrementado sobretodo en la población que comprende a niños, niñas, adolescentes y jóvenes menores de 25 años de sexo masculino, en particular entre los años 1990 y 2017 (Gerstner et al., 2019). 
Figura 7.

Causas de suicidios en la provincia de Tungurahua

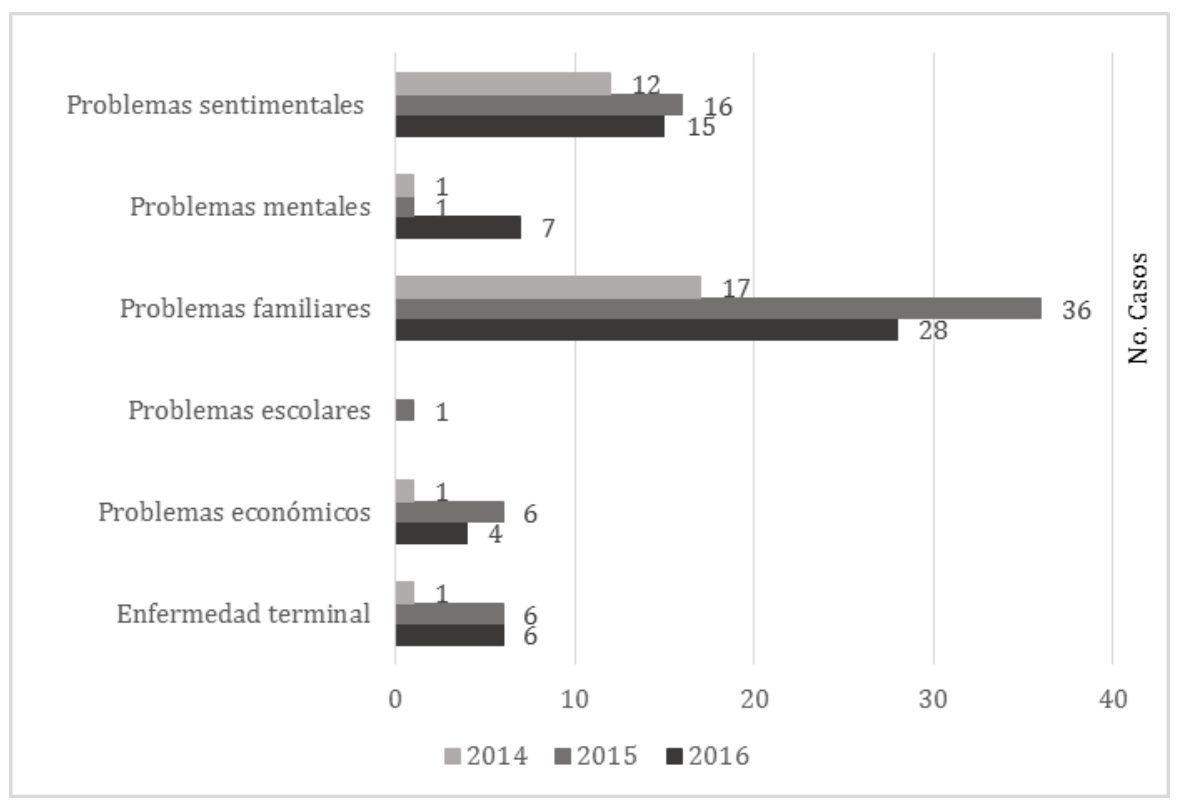

Fuente: Ministerio del Interior (2017)

Los problemas familiares representan las mayores estadísticas para el cometimiento de las lesiones autoinfligidas, en el año 2015 con treinta y seis casos, seguido del año 2016 en el que se contabilizan veinte y ocho suicidios por esta causa, como se puede ver en la Figura 7. Los problemas sentimentales se reportan como segunda causa de suicidios, siendo el 2015 el año en el que se reportan diez y seis casos. Los problemas mentales, los problemas escolares y los problemas económicos registran pocos casos en los suicidios por parte del Ministerio del Interior.

Las causas que provocan el suicidio se deben a diferentes factores, como problemas familiares, escolares, laborales, sentimentales, entre otros; estos a su vez se encuentran relacionados con situaciones emocionales que no pueden ser manejadas por el individuo, ocasionando que la persona tome su propia vida y se auto infrinja la muerte (Lafuente, \& Lema, 2020, p. 13). 
Figura 8.

Eventos de suicidios por días en la provincia de Tungurahua

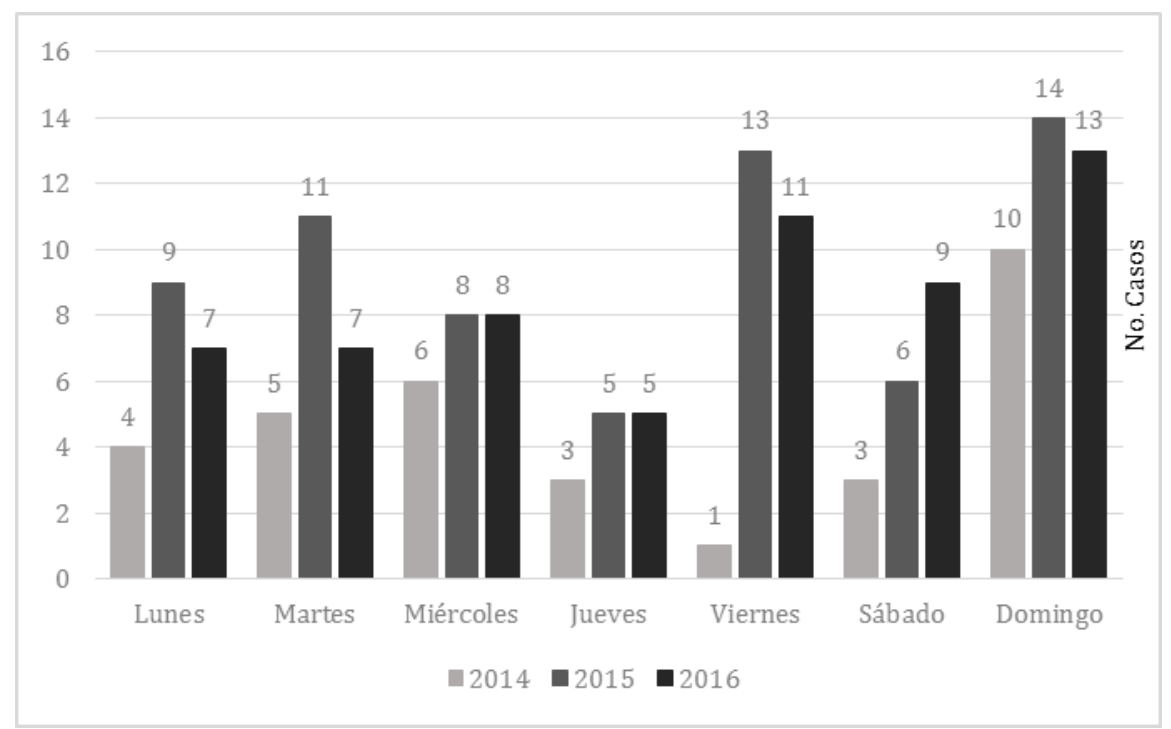

Fuente: Ministerio del Interior (2017)

En relación al día de la semana en el que se registró la mayor incidencia de suicidios, se evidencia que, en el día domingo es donde se presentan la mayor tasa de suicidios con treinta y siete casos, de los cuales el 6,33\% correspondió al año 2014 (Figura 8).

En el año 2015 se contabilizó un porcentaje de casos de suicidio correspondiente al 8,86\%; para el año 2016 del $8,21 \%$; de estos datos estadísticos los días que registraron altas tasas de suicidios fueron los viernes y martes con el $15,82 \%$ y $14,56 \%$ respectivamente. 
Figura 9.

Medios utilizados para el suicidio en la provincia de Tungurahua

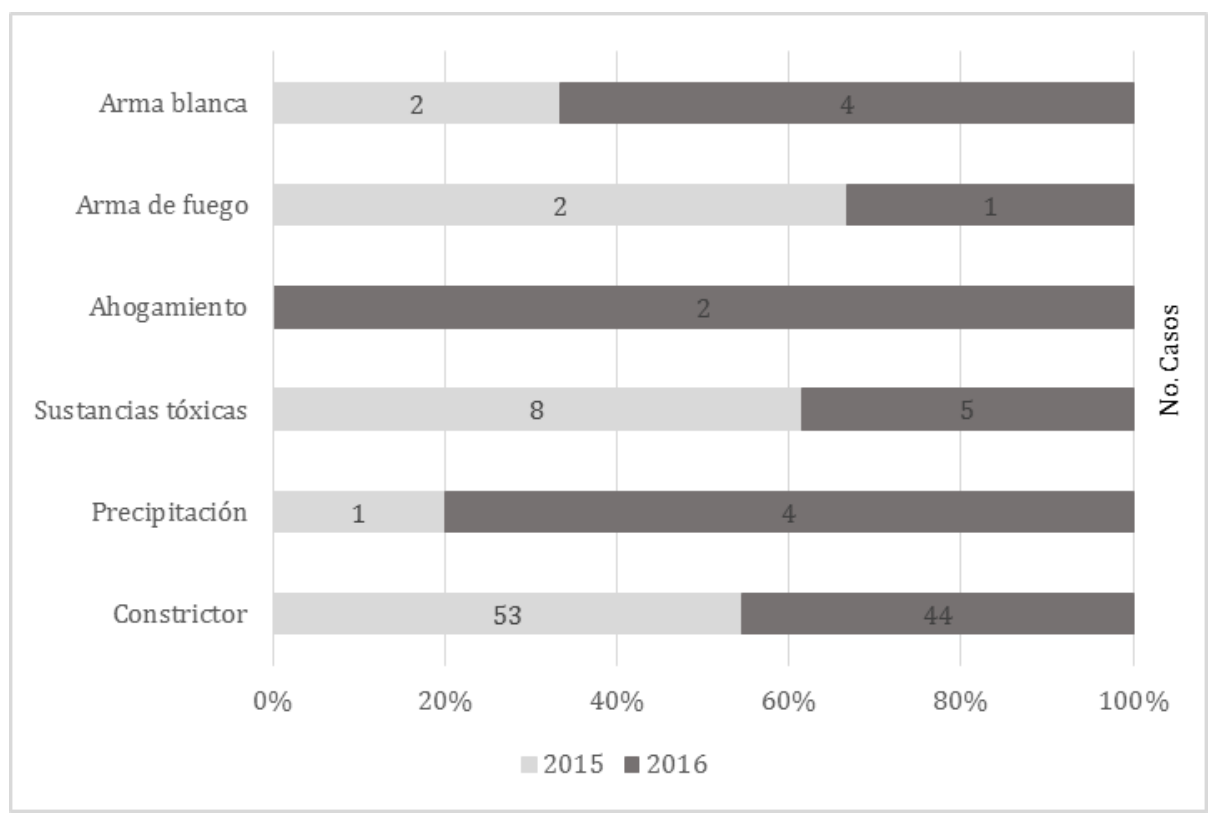

Fuente: Ministerio del Interior (2017)

En cuanto a los objetos constrictores como cuerdas, cables, correas, prendas textiles, etc., estos se constituyeron en el medio más utilizado por las personas en el cometimiento de suicidio. En este orden se registraron noventa y siete casos que equivalen al $76,26 \%$ del total de eventos reportados en la provincia de Tungurahua en los años 2015 y 2016.

En relación al consumo de sustancias toxicas y el uso de arma blanca que fueron los medios que se emplearon también con mayor frecuencia en el suicidio, estos representaron el $10,32 \%$ y $4,76 \%$ respectivamente, como se puede ver en la Figura 9.

En el siglo XX, entre los medios más comunes utilizados para cometer suicidio constituye el uso de arma blanca, manteniéndose más frecuente las ahorcaduras, las precipitaciones, los disparos, la sumersión y las intoxicaciones (Negre Muñoz \& Orozco Gómez, 2013, citado en Cazar, 2014, p. 49). 


\section{Figura 10.}

Total de suicidios en la provincia de Tungurahua

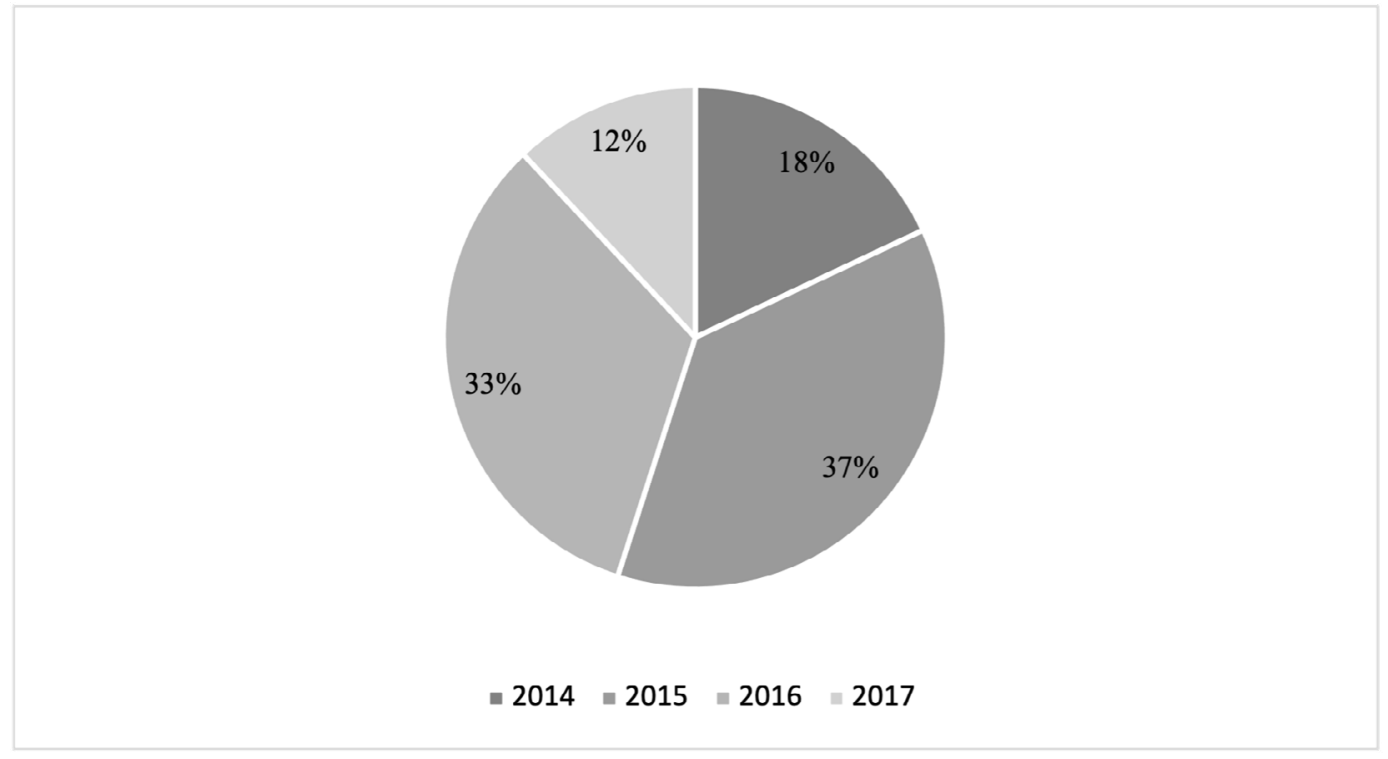

Fuente: Ministerio del Interior (Fecha de corte 31 mayo 2017)

En este sentido, de acuerdo con la Figura 10, se registraron ciento setenta y nueve suicidios en la provincia de Tungurahua que equivale al $10 \%$ de promedio en los cuatro años de estudio, siendo el año 2015 el periodo en el cual se produjo la mayor incidencia con el $37 \%$ de casos, seguido del año 2016 con el $33 \%$, que representan porcentajes considerables y preocupantes para ser tomados en consideración por el incremento permanente de casos año tras año. En el año 2014 se contabilizaron 32 suicidios representando el 18\%. Hasta el 31 de mayo del 2017 se registran 21 casos de eventos reportados que constituyen el $12 \%$ con tendencia a que se vayan aumentando los suicidios hasta finales de año.

\section{Conclusiones}

El suicidio en el Ecuador constituye la segunda causa de muerte entre adolescentes y adultos jóvenes, en edades comprendidas de 15 a 30 años. Desde el mes de marzo hasta noviembre del 2020, se registraron 220 suicidios (Universidad Tecnica Particular de Loja, 2021), afectando más a quienes ya venían con algún trastorno del estado de ánimo o de consumo de sustancias sujetas a fiscalización.

La Organización Mundial de la Salud hace una alerta preocupante en el 2020, en relación a la situación del suicidio en el Ecuador, debido a que se está incrementando paulatinamente en un $2 \%$ año tras año, exhortando a que el Estado realice de forma urgente programas eficientes de prevención y control, a través de campañas de concientización en donde se motive la participación activa de los municipios, gobiernos provinciales, instituciones educativas, y en general, a todos los actores sociales que puedan involucrarse y se conviertan en elementos multiplicadores para dar a conocer los principales factores de riesgo del suicidio. 
En la provincia de Tungurahua, no todos los casos de suicidio son reportados, en especial aquellos que se producen en estratos económicos altos, premisa que demarca zonificaciones de edad, género y los mecanismos utilizados en los hechos producidos en los sectores periférico y rurales, más en hombres que mujeres y hasta en los días de ejecución comprendidos en los fines de semana, como respuesta a celebraciones y consumo de alcohol.

Se refleja un índice elevado de casos de lesiones autoinflingidas intencionalmente por año en toda la provincia, en relación al número de población, siendo el año 2020 el que presenta la mayor tasa estadística de suicidios, de acuerdo a la Dirección Nacional de Delitos contra la Vida, Muertes Violentas, Desapariciones, Extorsión y Secuestros.

Los reportes estadísticos de lesiones autoinflingidas intencionalmente aparecen en grupos de edad que son muy temprano, por tal motivo se debe crear y fortalecer proyectos existentes como "Tú importas, elige vivir" dirigidos a los niños, a los jóvenes y personas en general.

Es importante fomentar la cultura de la buena convivencia sana y pacífica en los hogares ecuatorianos, ya que ahí radica uno de los principales detonantes del suicidio, debido a la crisis familiar existencial por la que atraviesan la mayoría de las familias, causadas por aspectos económicos, sociales, problemas con las relaciones paterno-filiales, problemas escolares.

Es fundamental coordinar acciones conjuntas entre los diferentes actores públicos como la Dirección Nacional de Delitos contra la Vida, Muertes Violentas, Desapariciones, Extorsión y Secuestros, el Ministerio de Salud Pública, Ministerio de Educación, el Ministerio de Inclusión Económica y Social, el Municipio de Ambato, la Gobernación de Tungurahua e instituciones privadas que coadyuven a la prevención oportuna y reducción del suicidio.

Finalmente, en la provincia de Tungurahua, el suicido debe ser tratado como un problema de atención pública, en procura de generar sensibilización a través de la familia para dar intervención desde sus causales, creando espacios que permitan al individuo hacer frente a este fenómeno, independientemente del estrato o nivel social de las personas, no obstante que la mayoría de casos reportados se relacionan a la clase media y baja. 


\section{Referencias}

Almeida, M. (2017). La campaña de minga por la vida de la gobernación de Tungurahua y la percepción de los lectores de los periódicos el Heraldo y la Hora del cantón Ambato. [Tesis de grado, Universidad Técnica de Ambato]. http://repositorio.uta.edu.ec/jspui/handle/123456789/25348

Bedoya, E., \& Montaño, L. (2016). Suicidio y Trastorno Mental (Suicide And Mental Disorder). Revista CES Psicolgía, 9(2), 179-201. https://doi.org/10.21615/cesp.9.2.12

Campo, A. (2018). Impacto del género en los intentos de suicidio en Quito (Ecuador). [Tesis doctorial, Universitat Rovira i Virgili]. TDX https://84.88.27.106/handle/10803/663365

Cazar, J. (2014). Incidencia y causas más comunes de suicidio en el periodo 2008 al 2012 en Pichincha, Quito-Ecuador. [Tesis de grado, Universidad de las Américas].

Cortés, A. (2019). Métodos y sustancias empleados en la conducta suicida en adolescentes. Revista Cubana de Medicina General Integral, 35(4) 1-14. http://www.revmgi.sld.cu/index.php/mgi/article/view/1105

Cuesta Revé, D. (2017). Aspectos epidemiológicos del suicidio en adolescentes. Revista Mexicana de Pediatría, (2), 72-77. https://www.medigraphic.com/cgi-bin/new/resumen.cgi?IDARTICULO=72271

DINASED. (2017). Dirección Nacional de Investigación de Delitos contra la Vida, Muertes Violentas, Desapariciones, Secuestros y Extorsión. Información proporcionada mediante entrevista a Roberto Llamuca, Jefe de la DINASED de Tungurahua.

Echávarri, O., Maino, M., Fischman, R., Morales, S., \& Barros, J. (2015). Aumento sostenido del suicidio en Chile: un tema pendiente. Centro de Políticas Públicas de la Pontificia Universidad Católica de Chile, 1-16.

Gerstner, R., \& Lara, F. (2019). Análisis de tendencias temporales del suicidio en niños, adolescentes y adultos jóvenes en Ecuador entre 1990 y 2017. An. Sist. Sanit. Navar, 42(1) 9-18. https://dx.doi.org/10.23938/ assn.0394

Gerstner, R.., Soriano, I., Sanhueza, A., Caffe, S., \& Kestel, D. (2018). Epidemiología del suicidio en adolescentes y jóvenes en Ecuador. Revista Panamericana de salud pública, 42, e100. https://doi.org/10.26633/ RPSP.2018.100

Instituto Nacional de Estadística y Censos. (2020). Estadísticas de Defunciones Generales en el Ecuador. INEC https://public.tableau.com/app/profile/instituto.nacional.de.estad.stica.y.censos.inec./viz/Registroestadsticodedefuncionesgenerales 15907230182570/Men 
Lafuente, B., \& Lema, A. (2020). Caracterización de muertes violentas en menores de edad en el Ecuador en el período 2012 - 2017. [Tesis de grado, Universidad Central del Ecuador] Repositorio UCE. http://www. dspace.uce.edu.ec/handle/25000/21417

Ministerio del Interior. (2017). Policía Nacional del Ecuador. Zona 3 Sub zona 18 Tungurahua. Información proporcionada mediante entrevista al Crnl. Rommel Navarrete Comandante de Policía de Tungurahua de la Sub zona 18.

Miniguano, G. (2018). Las relaciones familiares y los suicidios en el cantón Ambato provincia de Tungurahua. [Tesis de grado, Universidad Técnica de Ambato. http://repositorio.uta.edu.ec/jspui/handle/123456789/28055

Moreta, M. (3 de febrero de 2021). Autoridades de Tungurahua trabajan en una campaña para bajar índices de suicidio. El Comercio. https://www.elcomercio.com/actualidad/ecuador/tungurahua-campana-indices-suicidio-muerte.html

Organización Mundial de la Salud. (17 de junio de 2021). Suicidio. OMS. https://www.who.int/es/news-room/ fact-sheets/detail/suicide

Organización Panamericana de la Salud. (2014). Prevención del suicidio: un imperativo global. PAHO. https:// iris.paho.org/handle/10665.2/54141

Pérez, E. (Julio de 2019). Violencia de género como factor de riesgo del suicidio. [Tesis de grado, Universidad de Almería].

Salgado, B. (30 de octubre de 2020). Dinased presentó cifras de suicidios y formas de prevención. Policía Nacional del Ecuador https://www.policia.gob.ec/dinased-presento-cifras-de-suicidios-y-formas-de-prevencion/ 
Copyright (c) 2022 Jorge Oswaldo Tamayo Viera, Vicente Leonardo Pérez Yauli, Ibeth Aracelly Molina Arcos y Edison Manuel Arroba Freire

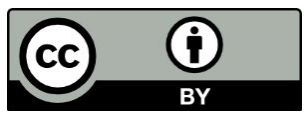

Este texto está protegido bajo una licencia internacional Creative Commons 4.0.

Usted es libre para Compartir-copiar y redistribuir el material en cualquier medio o formato

- y Adaptar el documento - remezclar, transformar y crear a partir del material-para cualquier propósito, incluso para fines comerciales, siempre que cumpla las condiciones de Atribución. Usted debe dar crédito a la obra original de manera adecuada, proporcionar un enlace a la licencia, e indicar si se han realizado cambios. Puede hacerlo en cualquier forma razonable, pero no de forma tal que sugiera que tiene el apoyo del licenciante o lo recibe por el uso que hace de la obra.

\section{$\underline{\text { Resumen de licencia - Texto completo de la licencia }}$}

